\title{
Solo and Keratin Filaments Regulate Epithelial Tubule Morphology
}

\author{
Ryosuke Nishimura ${ }^{1,2}$, Kagayaki Kato ${ }^{3,4,5}$, Sachiko Fujiwara ${ }^{6}$, Kazumasa Ohashi ${ }^{1^{*}}$, and Kensaku Mizuno ${ }^{1^{* *}}$ \\ ${ }^{1}$ Department of Biomolecular Sciences, Graduate School of Life Sciences, Tohoku University, 6-3, Aramaki- \\ Aoba, Aoba-ku, Sendai, Miyagi 980-8578, Japan, ${ }^{2}$ Department of Cell Biology, Graduate School of Medical \\ Sciences, Tokushima University, 3-18-15, Kuramoto-cho, Tokushima, Tokushima 770-8503, Japan, ${ }^{3}$ Bioimage \\ Informatics Group, Exploratory Research Center on Life and Living Systems (ExCELLS), National Institutes \\ of Natural Sciences (NINS), 38 Nishigonaka, Myodaiji, Okazaki, Aichi 444-8585, Japan, ${ }^{4}$ Department of \\ Imaging Science, Center for Novel Science Initiatives (CNSI), National Institutes of Natural Sciences (NINS), \\ 38, Nishigonaka, Myodaiji, Okazaki, Aichi 444-8585, Japan, ${ }^{5}$ Division of Evolutionary Biology Biodiversity, \\ National Institute for Basic Biology (NIBB), 38, Nishigonaka, Myodaiji, Okazaki, Aichi 444-8585, Japan, \\ ${ }^{6}$ Division of Bioengineering, Graduate School of Engineering Science, Osaka University, 1-3, \\ Machikaneyama-chou, Toyonaka, Osaka 560-8531, Japan
}

\begin{abstract}
Epithelial tubules, consisting of the epithelial cell sheet with a central lumen, are the basic structure of many organs. Mechanical forces play an important role in epithelial tubulogenesis; however, little is known about the mechanisms controlling the mechanical forces during epithelial tubule morphogenesis. Solo (also known as ARHGEF40) is a RhoA-targeting guanine-nucleotide exchange factor that is involved in mechanical forceinduced RhoA activation and stress fiber formation. Solo binds to keratin-8/keratin-18 (K8/K18) filaments, and this interaction plays a crucial role in mechanotransduction. In this study, we examined the roles of Solo and K8/K18 filaments in epithelial tubulogenesis using MDCK cells cultured in 3D collagen gels. Knockdown of either Solo or K18 resulted in rounder tubules with increased lumen size, indicating that Solo and K8/K18 filaments play critical roles in forming the elongated morphology of epithelial tubules. Moreover, knockdown of Solo or K18 decreased the level of diphosphorylated myosin light chain (a marker of contractile force) at the luminal and outer surfaces of tubules, suggesting that Solo and K8/K18 filaments are involved in the generation of the myosin IImediated contractile force during epithelial tubule morphogenesis. In addition, K18 filaments were normally oriented along the long axis of the tubule, but knockdown of Solo perturbed their orientation. These results suggest that Solo plays crucial roles in forming the elongated morphology of epithelial tubules and in regulating myosin II activity and K18 filament organization during epithelial tubule formation.
\end{abstract}

Key words: epithelial tubulogenesis, Solo, keratin, Rho-GEF, myosin

\footnotetext{
*To whom correspondence should be addressed: Kazumasa Ohashi, Department of Biomolecular Sciences, Graduate School of Life Sciences, Tohoku University, Sendai, Miyagi 980-8578, Japan.

Tel: +81-22-795-6590, Fax: +81-22-795-6594

E-mail: kazumasa.ohashi.b2@tohoku.ac.jp

**To whom correspondence should be addressed: Kensaku Mizuno, Department of Biomolecular Sciences, Graduate School of Life Sciences, Tohoku University, Sendai, Miyagi 980-8578, Japan.

Tel: +81-22-795-6590, Fax: +81-22-795-6594

E-mail:kmizuno@biology.tohoku.ac.jp

Abbreviations: 3D, three-dimensional; DAPI, 4',6-diamidino-2phenylindol; DMEM, Dulbecco's modified Eagle medium; ECM, extracellular matrix; FBS, fetal bovine serum; GAPDH, glyceraldehyde-3phosphate dehydrogenase; GEF, guanine-nucleotide exchange factors; GFP, green fluorescent protein; HGF, hepatocyte growth factor; K8, keratin-8; K18, keratin-18; MDCK, Madin-Darby canine kidney; MLC, myosin light chain 2; PBS, phosphate-buffered saline; ppMLC, Thr18, Ser19-diphosphorylated myosin light chain 2; siRNA, small interfering RNA; YFP, yellow fluorescent protein.
}

\section{Introduction}

Hollow tubes are the basic structure of many organs, such as the kidney, lung, liver, gastrointestinal tract, blood vessels, and exocrine glands. Epithelial tubules consist of a single lumen surrounded by a layer of epithelial cells and their formation requires the coordination of many cellular processes including cell migration, morphogenesis, polarity formation, proliferation, and apoptosis (Lubarsky and Krasnow, 2003). While a variety of molecules, including cell adhesion molecules, cytoskeletal components, and growth factors, are involved in the process of tubule formation, mechanical forces also play a crucial role in tubulogenesis (Wozniak and Chen, 2009; Iruela-Arispe and Beitel, 2013). Madin-Darby canine kidney (MDCK) epithelial cells cultured in three-dimensional (3D) gels have been 
frequently used as a model system to investigate mechanisms of epithelial tubule formation (Pollack et al., 1998; O'Brien et al., 2002; Zegers et al., 2003; Sigurbjornsdottir et al., 2014). When MDCK cells are cultured in 3D collagen gels or Matrigel, they first form the cyst which is a spherical structure consisting of a monolayer of polarized cells with a central lumen. Stimulation of the cyst with hepatocyte growth factor (HGF) induces the formation of an elongated tubular structure through a series of morphogenetic processes (Montesano et al., 1991a; Montesano et al., 1991b; O'Brien et al., 2002). Recent studies reveal the important roles of cytoskeletal remodeling, mechanotransduction, and membrane transport, as well as cell migration, adhesion, and polarization, in epithelial tubule formation (Miao et al., 2003; Hunter and Zegers, 2010; Kim et al., 2010; Harada et al., 2015; Kim et al., 2015; Marty-Santos and Cleaver, 2016). However, the molecular mechanism that regulates the morphology and lumen size of epithelial tubules remains poorly understood.

Rho family small GTPases are key regulators of actin cytoskeletal remodeling (Hall, 2012). Rho family proteins play important roles in numerous cellular events, including migration, morphogenesis, adhesion, polarization, and mechanotransduction (Geiger et al., 2009; Huveneers and de Rooij, 2013; Ohashi et al., 2017); thus, they appear to be crucial for tubular morphogenesis. Rho family proteins are activated by guanine-nucleotide exchange factors (GEFs) that promote the conversion of Rho family GTPases from inactive GDP-bound forms to active GTP-bound forms. The human genome possesses approximately 70 genes encoding Dbl-like Rho-GEFs, each of which appears to activate a specific set of Rho family proteins (Cook et al., 2014; Hodge and Ridley, 2016). Recently, we showed that Solo (also known as ARHGEF40), a RhoA-targeting GEF (Curtis et al., 2004; Tse et al., 2005), is involved in cyclicstretch-induced reorientation of vascular endothelial cells (Abiko et al., 2015). We have also shown that Solo binds to keratin-8/keratin-18 (K8/K18) filaments and that the interaction between Solo and K8/K18 filaments is crucial for tensional force-induced RhoA activation and stress fiber reinforcement in epithelial cells (Fujiwara et al., 2016).

Keratin filaments are stable but resilient cytoskeletal filaments that provide mechanical support for epithelial cells and tissues (Coulombe and Wong, 2004; Fujiwara and Mizuno, 2017). K8/K18 filaments, composed of heteropolymers of $\mathrm{K} 8$ and $\mathrm{K} 18$, are the major intermediate filaments in simple epithelial cells. K8/K18 filaments mediate the extracellular matrix $(\mathrm{ECM})$ rigidity-induced increase in cell stiffness through the RhoA-ROCK pathway (Bordeleau et al., 2012). We recently showed that K8/K 18 filaments are required for tensional force-induced RhoA activation and stress fiber formation in MDCK cells (Fujiwara et al., 2016). These findings prompted us to investigate the roles of Solo and K8/K18 filaments in epithelial tubule morphogenesis.
In this study, using MDCK cells cultured in 3D collagen gels as a model system of epithelial tubule formation, we demonstrate that Solo and K8/K18 filaments are crucial for regulating the elongated morphology of epithelial tubules.

\section{Materials and Methods}

\section{Reagents and antibodies}

Rabbit polyclonal antibodies against human Solo, which also recognized dog Solo, were prepared as described previously (Abiko et al., 2015). Polyclonal antibodies against green fluorescent protein (GFP) (A6455; Thermo Fisher Scientific), myosin light chain 2 (MLC) (3672; Cell Signaling), and Thr18/Ser19diphosphorylated myosin light chain 2 (ppMLC) (3674S; Cell Signaling), and monoclonal antibodies against K18 (Ks 18.04; Progen), $\beta$-actin (AC-15; Sigma-Aldrich), GFP (RQ2; MBL), glyceraldehyde-3-phosphate dehydrogenase (GAPDH) (14C10; Cell Signaling), and $\alpha$-tubulin (ab56676; Abcam) were purchased from the indicated suppliers. Collagen (type I, BD Biosciences), HGF (PeproTech), Rhodamine-labeled phalloidin (Wako Pure Chemical Industries), Alexa Fluor 350-labeled phalloidin (Thermo Fisher Scientific), and 4',6-diamidino-2-phenylindol (DAPI, Polysciences) were purchased from the indicated suppliers.

\section{Plasmid construction and siRNAs}

The expression plasmid encoding yellow fluorescent protein (YFP)-tagged K8 was constructed as described previously (Fujiwara et al., 2016). The small interfering RNAs (siRNAs) targeting dog Solo and K18 were purchased from Sigma-Aldrich. The siRNA targeting sequences were as follows: 5'-GAG CTG AAA GAG GAA CTC AAA CC-3' (Solo siRNA \#1), 5'-GGG ATC AGA GAC CTT TGT TTA CA-3' (Solo siRNA \#2), 5'-GAG TTG GAT GCC CCC AAA TCT CA-3' (K18 siRNA \#1), and 5'AGG CGT TGA GGC AGC AAA ACA GG-3' (K18 siRNA \#2). MISSON siRNA Universal Negative Control (SIC001, SigmaAldrich) was used as the negative control for siRNA transfections.

\section{Cell culture and siRNA transfection}

MDCK cells were cultured in Dulbecco's modified Eagle medium (DMEM) containing 10\% fetal bovine serum (FBS). MDCK cell lines stably expressing YFP-Lifeact were established as previously described (Fujiwara et al., 2016). MDCK cell lines stably expressing YFP-K18 were established using G418-containing medium for selection. Cells were transfected with siRNAs using Lipofectamine RNAiMAX (Life Technologies). Typically, cells were plated in $35-\mathrm{mm}$ culture dishes at a density of $4.0 \times 10^{4}$ cells/ dish, cultured for $24 \mathrm{~h}$, and then transfected with siRNAs. After $24 \mathrm{~h}$, cells were harvested and subjected to 3D culture in collagen gels. The siRNAs were used at a final concentration of $20 \mathrm{nM}$ (for Solo knockdown) or $50 \mathrm{nM}$ (for K18 knockdown). 


\section{Tubule formation of MDCK cells in 3D collagen gels}

For tubule formation assays, MDCK cells were cultured at the interface between stiff and soft collagen gels. To prepare the stiff collagen gel, the stock collagen solution was diluted with DMEM to a final concentration of $2 \mathrm{mg} / \mathrm{mL}$ collagen, and aliquots (100 $\mu \mathrm{L})$ were dispensed into wells of 48 -well plates and allowed to form gels by incubating for $>30 \mathrm{~min}$ at $37^{\circ} \mathrm{C}$. MDCK cells harvested from $2 \mathrm{D}$ culture using trypsin were suspended at a concentration of $5 \times 10^{3}$ cells $/ \mathrm{mL}$ in DMEM containing $0.1 \mathrm{mg} / \mathrm{mL}$ collagen (soft collagen) and $10 \mathrm{ng} / \mathrm{mL} \mathrm{HGF}$, and aliquots $(200 \mu \mathrm{L})$ of the cell suspension were loaded on top of the stiff collagen gel in wells of 48-well plates. Cells settled onto the surface of the stiff collagen gel before gelation of the soft collagen solution. Cells were cultured for 4 days. Half $(100 \mu \mathrm{L})$ of the upper gel was renewed 2 days after cell loading.

\section{Immunoblot analysis}

To determine siRNA knockdown efficiencies, cells were transfected with siRNAs, cultured for 5 days, and lysed with SDS sample buffer containing 1\% SDS. Cell lysates were separated with SDSPAGE and subjected to immunoblot analysis as described previously (Abiko et al., 2015). The membranes were analyzed using antibodies against Solo, K18, $\beta$-actin, and $\alpha$-tubulin. To examine the effects of Solo or K18 knockdown on the levels of ppMLC and MLC, MDCK cells were transfected with siRNAs and cultured on plates for 2 days, and the cell lysates were analyzed by immunoblotting using antibodies against MLC and ppMLC. To examine the effect of Solo knockdown on the level of YFP-K18, MDCK cells stably expressing YFP-K18 were transfected with Solo siRNAs and cultured on plates for 2 days, and the cell lysates were analyzed by immunoblotting using anti-GFP (RQ2) and antiGAPDH antibodies.

\section{Immunostaining and fluorescence imaging}

For immunofluorescence staining except for ppMLC, cells cultured in collagen gels were rinsed once with phosphate-buffered saline (PBS), fixed with 4\% paraformaldehyde for $20 \mathrm{~min}$, and then permeabilized with $0.5 \%$ Triton X-100 in PBS for $5 \mathrm{~min}$. Tubules were washed three times with PBS for $10 \mathrm{~min}$ and incubated with $2 \%$ FBS to reduce non-specific binding of antibodies. They were incubated with primary antibodies over night at $4{ }^{\circ} \mathrm{C}$, washed three times with PBS for $10 \mathrm{~min}$, incubated with secondary antibodies or fluorescent reagents (Rhodamine- or AlexaFluor-350-labeled-phalloidin, or DAPI) for $30 \mathrm{~min}$, and washed three times with PBS for $10 \mathrm{~min}$. Collagen gels containing cells were removed from the wells of 48 -well dishes and mounted with mounting buffer $(90 \%$ glycerol and $0.1 \%$ paraphenylenediamine in PBS) on $35-\mathrm{mm}$ glass bottom dishes. For staining ppMLC, cells were fixed and permeabilized with $4 \%$ paraformaldehyde and $0.5 \%$ Triton $\mathrm{X}-100$ in PBS (warmed at $37^{\circ} \mathrm{C}$ in advance) for $20 \mathrm{~min}$. Cells were washed twice with PBS for $5 \mathrm{~min}$, three times with Tris-buffered saline for $5 \mathrm{~min}$, and incubated with blocking buffer (10\% FBS and 1\% bovine serum albumin in PBS) for 20 min. Then, cells were incubated with a primary antibody for ppMLC (Bhadriraju et al., 2007) for $45 \mathrm{~min}$, washed five times with $0.05 \%$ Triton $\mathrm{X}-100$ in PBS for $10 \mathrm{~min}$, incubated with blocking buffer for $10 \mathrm{~min}$ and secondary antibody for $45 \mathrm{~min}$, washed five times, and mounted as described above. Fluorescence images were obtained using an LSM510 or LSM710 laser scanning confocal microscope (Carl Zeiss Microscopy) equipped with a PL Apo 100× oil objective lens (NA 1.4) or a PL Apo 40× objective lens (NA 1.3).

\section{Image analysis of tubule and lumen morphology}

Images were processed and analyzed using ImageJ software (National Institutes of Health) or CellProfiler software (Carpenter et al., 2006). To measure tubule morphology, 3D confocal stack images of tubules were captured, where the $x-y$ view was set to have the largest cross-sectional area of the tubule. The outlines of whole tubule and lumen in the $\mathrm{x}-\mathrm{y}$ cross-sections were traced freehand, and the ratio of the luminal area to the total (lumen plus cells) area was calculated. Tubule roundness was analyzed by fitting the outline of the $x-y$ cross-section of each tubule to an ellipse and measuring the ratio of the length of the short to that of the long axis. The cell number in each tubule was analyzed by counting the number of nuclei in the tubule. The nuclei of cells in tubules were stained with DAPI or TO-PRO-3 and the Z-stacked images were captured by a confocal microscopy. An interval for the z-axis of the image stack was interpolated by the Lanczos- 5 resampling algorithm to fit it to the $\mathrm{x}$ - and y-resolutions. To emphasize individual nuclei in this stack, a Difference of Gaussian (DoG) filter was applied to its optimal sizes for nuclei radii (Azuma and Onami, 2013), and nuclei were identified by scanning intensity peaks throughout the stack. The intensity peaks outside the tubule were eliminated by scanning only within the tubule region that was obtained by $\mathrm{F}$-actin staining. These procedures were implemented in $\mathrm{C}$ and Ruby to build an automated pipeline for processing over 1,000 confocal image stacks.

\section{Image analysis of K18 filaments, F-actin, and ppMLC}

To measure the orientation of K18 filaments, the outline of each tubule was traced freehand and fitted to an ellipsoid, and the longest ( $\mathrm{x}$ ) axis of the ellipsoid was defined. Then, $\mathrm{z}$-stacked images of YFP-K18 were processed with the enhancing method and converted to binary images by auto-thresholding. The angle $(\theta)$ of the orientation of K18 filaments against the longest (x) axis of the ellipsoid was measured. Mean fluorescence intensity of K18 filaments was measured from the z-stacked image of YFP-K18 in the tubule outline. To measure the fluorescence intensities of F-actin and ppMLC, the tubules were fixed and stained with Alexa Fluor 350-phalloidin and an anti-ppMLC antibody and analyzed by line profiles of their fluorescence intensities in the $x-y$ cross-section of tubules. Maximum fluorescence intensities on lines crossing the apical and the basal region of each cell were measured and the mean values were calculated (e.g., if 20 cells were present in the 
$\mathrm{x}-\mathrm{y}$ plane, the fluorescence intensities of F-actin and ppMLC on the apical and the basal region of each cell were analyzed using 20 line profiles).

\section{Statistical analyses}

Data are expressed as means \pm SD of three to four independent experiments. All statistical analyses were performed in Prism 6 (GraphPad Software). P-values were calculated using one-way ANOVA followed by Dunnett's test for multiple dataset comparisons. In all cases, $P<0.05$ was considered statistically significant.

\section{Results}

\section{Knockdown of Solo leads to the formation of rounder epithelial tubules with increased lumen size}

To examine the role of Solo in epithelial tubule formation and morphogenesis, MDCK cells stably expressing YFPLifeact (as a marker to visualize actin filaments) were cultured in 3D collagen gels as described in Materials and Methods. Briefly, MDCK cells were suspended in the growth medium containing $0.1 \mathrm{mg} / \mathrm{mL}$ collagen (soft collagen) and HGF, and loaded onto the stiff collagen gel containing $2 \mathrm{mg} / \mathrm{mL}$ collagen (Fig. 1A). Long, oval, closed-end tubular structures were formed at the interface between soft and stiff collagen gels 4 days after 3D culture. Tubules were fixed at 4 days because most tubules grew larger than the visual field of the $40 \times$ objective lens of a confocal microscope if they were cultured for more than 4 days. Tubule morphology was analyzed by confocal microscopic imaging of YFP-Lifeact fluorescence (Fig. 1B). To examine the role of Solo in tubule formation, MDCK cells stably expressing YFP-Lifeact were treated with control or Solotargeting siRNAs. Two independent siRNAs targeting Solo suppressed the expression of endogenous Solo protein in MDCK cells (Fig. 1C). Similar to control siRNA-treated cells, Solo-knockdown cells formed a closed-end tubular structure with a single lumen and exhibited accumulation of actin filaments in the luminal (apical) region of tubules (Fig. 1B), suggesting that Solo knockdown had no obvious effect on single lumen formation and apico-basal tubule polarity. Overall, the size of whole tubules did not differ between control and Solo-knockdown tubules, but Soloknockdown tubules were rounder than control tubules (Fig. 1B).

To quantitatively measure the effect of Solo knockdown on tubule morphology, we selected $>50$ similarly sized control and Solo-knockdown tubules and captured their 3D confocal stack images, where the $\mathrm{x}-\mathrm{y}$ view was set to have the largest cross-sectional area of the tubule (Fig. 1B, D, and Supplementary Videos S1 and S2). Because we selected similarly sized tubules, the total (lumen plus cell) area of the $x-y$ cross-section was similar between Solo- knockdown tubules and control tubules (Fig. 1E). The zstack image analysis of the whole tubule revealed that Solo knockdown had no apparent effect on the total number of cells in similarly sized tubules (Fig. 1F). We next analyzed the morphology of tubules by fitting the outline of the $x-y$ cross-section of each tubule to an ellipse (Fig. 1D) and measuring the ratio of the length of the short axis to the long axis. This ratio was significantly higher for the tubules formed by Solo-knockdown cells than for the tubules formed by control cells (Fig. 1G), indicating that Solo knockdown resulted in a rounder tubule morphology. We also analyzed the effect of Solo knockdown on tubule lumen size. Knockdown of Solo significantly increased the ratio of the luminal area to the total (lumen plus cell) area of the $\mathrm{x}-\mathrm{y}$ cross-section of tubules (Fig. 1H). These results indicate that Solo knockdown increases the roundness and lumen size of epithelial tubules and that Solo is involved in the formation or maintenance of properly elongated morphology of epithelial tubules. Because overexpression of Solo impaired the ability of MDCK cells to form tubules, it was difficult to perform the knockdown-rescue experiments for the effect of Solo siRNAs on tubule formation. In this regard, we previously showed that the inhibitory effects of Solo siRNAs on tensional force-induced stress fiber formation were rescued by expression of Solo in MDCK cells (Fujiwara et al., 2016), which suggests that the effects of Solo siRNAs used in this study are the specific effects of Solo knockdown.

\section{Knockdown of $K 18$ results in rounder epithelial tubules with increased lumen size}

We previously showed that Solo binds to K8/K18 filaments and their interaction is required for tensional force-induced RhoA activation and stress fiber formation (Fujiwara et al., 2016). To examine the role of $\mathrm{K} 8 / \mathrm{K} 18$ filaments in epithelial tubule morphogenesis, we tested the effect of K18 knockdown on the morphology of 3D-cultured MDCK tubules. Two K18-targeting siRNAs effectively suppressed the expression of K18 protein in MDCK cells (Fig. 2A). The K18-knockdown cells formed tubules with a single lumen and accumulated actin filaments in the luminal region of the tubules (Fig. 2B). Similar to the results observed in Solo-knockdown cells, the overall tubule size did not differ between control and K18-knockdown cells, but K18-knockdown tubules appeared to be rounder than those formed by control cells (Fig. 2B). We selected $>50$ similarly sized tubules of control and K18-knockdown cells (Fig. 2C). Quantitative analyses revealed that knockdown of K18 had no significant effect on the total number of cells per tubule (Fig. 2D). However, the ratio of the length of the short to the long axis of tubules (Fig. 2E) and the ratio of the luminal area to the total area in the $\mathrm{x}-\mathrm{y}$ cross-section of tubules (Fig. 2F) were significantly higher in tubules formed by K18-knockdown cells than in those formed by 
A
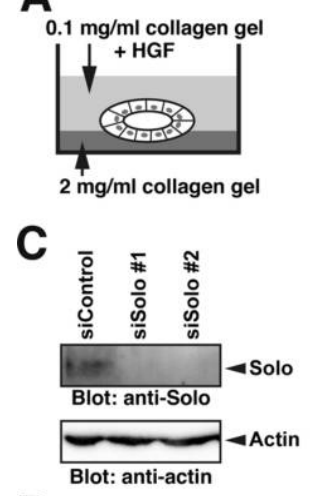

D
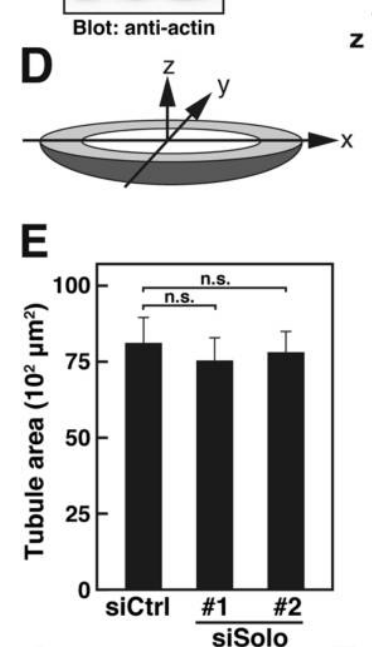

B
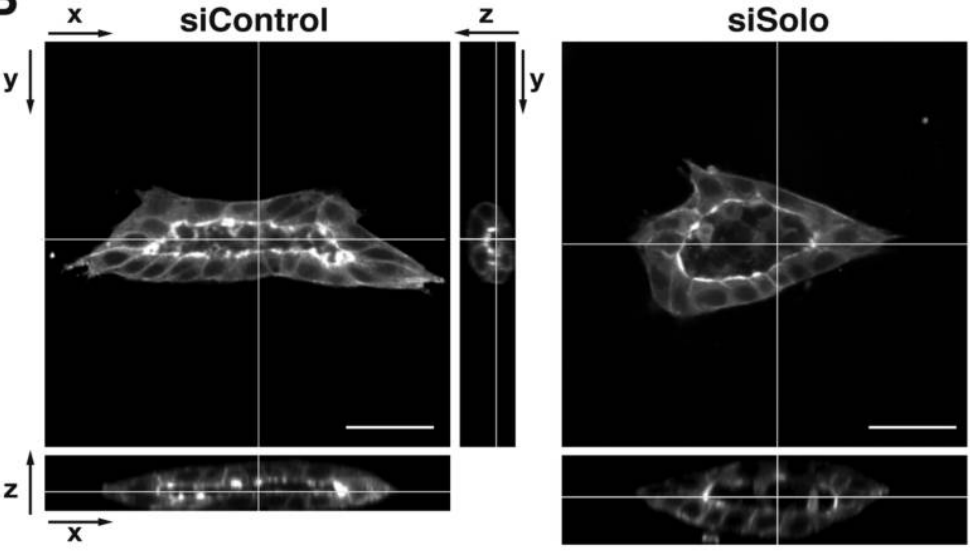

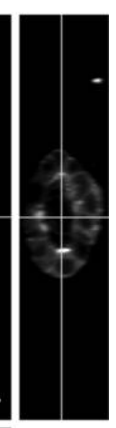

$\mathbf{F}$

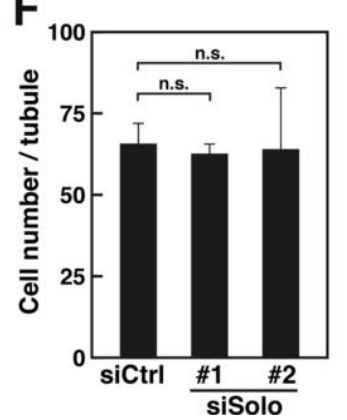

G

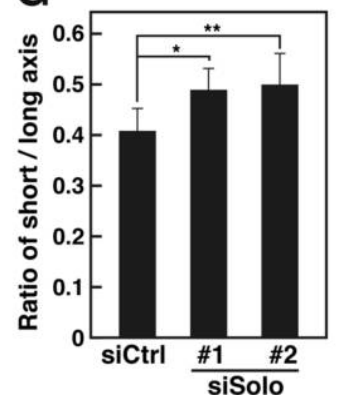

H

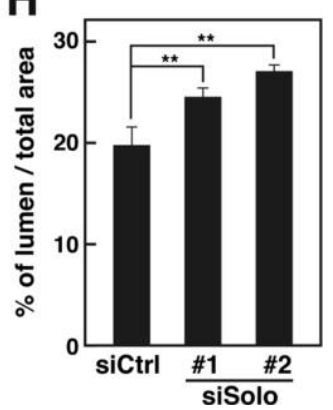

Fig. 1. Knockdown of Solo results in epithelial tubules with rounder morphology and wider lumen. (A) Schematic diagram of the 3D culture system used in this study. (B) Representative confocal microscopic images of YFP-Lifeact (F-actin) of MDCK tubules. MDCK cells stably expressing YFP-Lifeact were transfected with either control or Solo-targeting siRNAs, cultured for $24 \mathrm{~h}$, and then cultured in 3D collagen gels in the presence of HGF for 4 days. Cells were fixed and YFP fluorescence was detected. The $x-z$ and $y-z$ views were taken at the lines indicated in the $x-y$ view. Scale bar, $50 \mu \mathrm{m}$. (C) Effects of Solo siRNAs on Solo expression. MDCK cells transfected with either control or Solo-targeting siRNAs were cultured for 5 days. Cell lysates were analyzed by immunoblotting with antibodies against Solo and $\beta$-actin. (D) Schematic 3D image of the closed-end tubule. (E) Comparison of the $x$-y crosssectional area between control and Solo-knockdown tubules. The x-y axial section was set to have the largest cross-sectional area of the tubule. (F) Effect of Solo knockdown on the total cell number in each tubule. The total cell number was analyzed as the total number of nuclei in the z-stack image of each tubule. (G) Effect of Solo knockdown on tubule roundness. The roundness was analyzed by fitting the x-y cross-section of the tubule to an ellipse and measuring the ratio of the length of the short axis to that of the long axis. $(\mathrm{H})$ Effect of Solo knockdown on the luminal size of tubules. The percentage of the luminal to the total (luminal+cells) area of the $\mathrm{x}-\mathrm{y}$ cross-section of the tubule was calculated. In $(\mathrm{E})-(\mathrm{H})$, data represent mean $\pm \mathrm{SD}$ from three independent experiments (at least 50 tubules per experiment). ${ }^{*} P<0.05 ; * * P<0.01$; n.s., not significant (one-way ANOVA followed by Dunnett's test).

control cells. These results suggest that similar to Solo, $\mathrm{K} 18$ is involved in the formation or maintenance of the elongated morphology of epithelial tubules.

\section{Knockdown of Solo or K18 decreases the level of ppMLC at the luminal and the basal regions of tubules}

The myosin II-mediated contractile force seems to play an important role in tubule morphogenesis (Recuenco et al., 2015). Because Solo and K8/K18 filaments are involved in mechanical force-induced RhoA activation (Fujiwara et al., 2016) and the RhoA-ROCK pathway stimulates myosin IImediated contractility (Fukata et al., 2001), we hypothe- sized that Solo and K8/K18 filaments are involved in the regulation of myosin II-mediated contractile force during tubule formation. We therefore examined the effects of Solo or K18 knockdown on the level of ppMLC, which is widely used as a marker of contractile force in cells (Goeckeler and Wysolmerski, 1995; Mizutani et al., 2006), in MDCK tubules. MDCK cells were transfected with either control or Solo-targeting siRNAs and cultured in 3D collagen gels for 4 days, and then fixed and co-stained with an anti-ppMLC antibody and Alexa Fluor-350-labeled phalloidin to detect F-actin. The ppMLC signal accumulated in both the luminal (apical) and the outer (basal) regions of tubules in control cells, where the signal in the former 
A

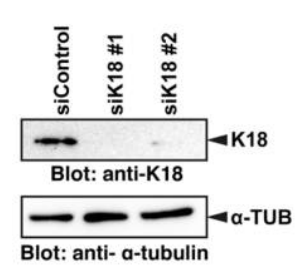

C

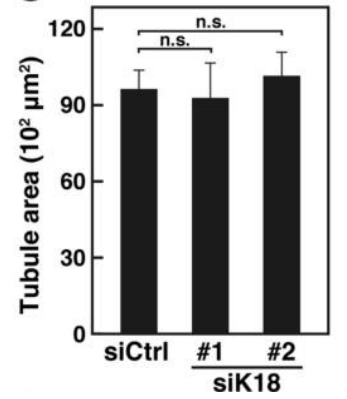

B

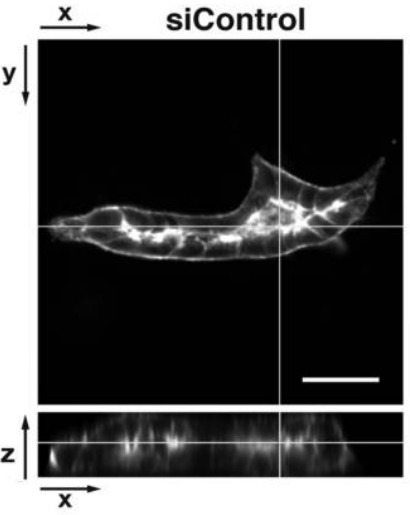

D

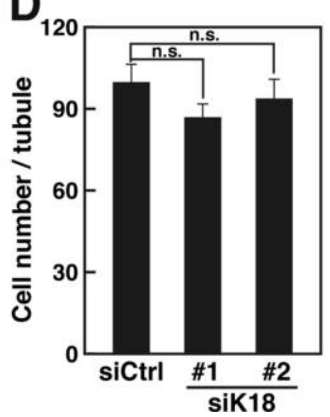

E
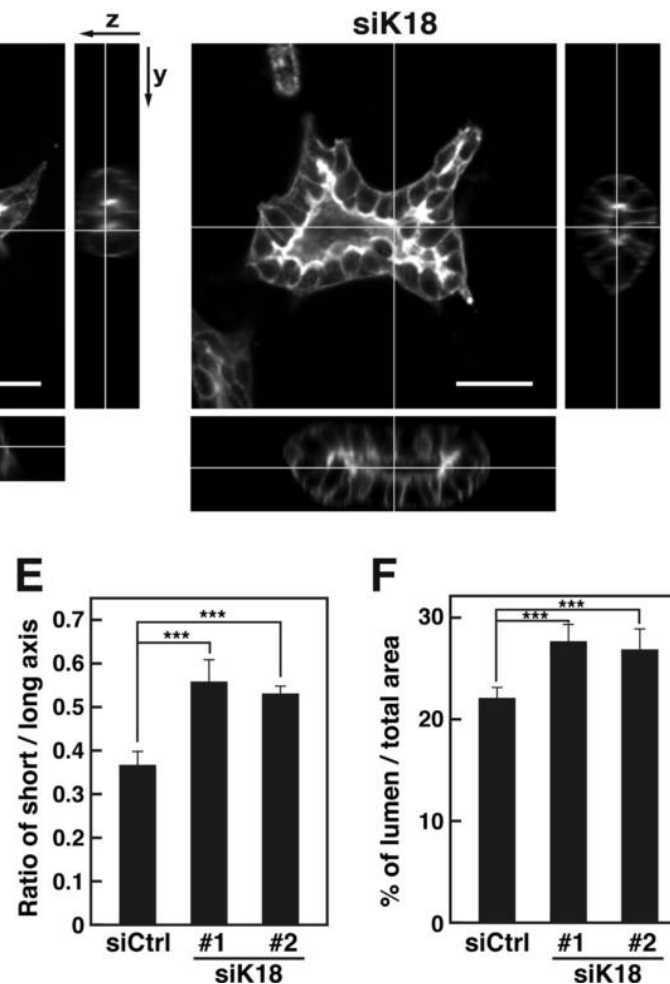

F

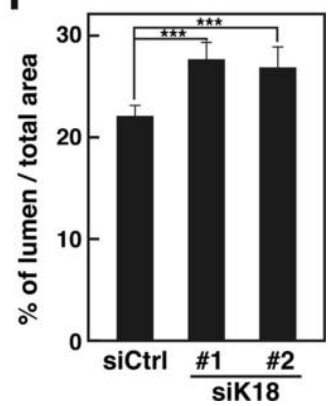

Fig. 2. Knockdown of K18 results in epithelial cells with rounder morphology and wider lumen. (A) Effects of K18-targeting siRNAs on the expression of K18. MDCK cells transfected with either control or K18-targeting siRNAs were cultured for 5 days and cell lysates were analyzed by immunoblotting with antibodies against K18 and $\alpha$-tubulin. (B) Representative images of F-actin of control and K18-knockdown tubules. MDCK cells transfected with either control or K18 siRNAs were cultured in 3D collagen gels, as shown in Fig. 1B. Cells were fixed and stained with rhodamine-phalloidin. The X-Z and $\mathrm{y}-\mathrm{z}$ views were taken at the lines indicated in the $\mathrm{x}-\mathrm{y}$ views. Scale bar, $50 \mu \mathrm{m}$. (C) Comparison of the $\mathrm{x}-\mathrm{y}$ cross-sectional area between control and K18knockdown tubules. (D) Effect of K18 knockdown on the total cell number in each tubule. (E) Effect of K18 knockdown on tubule roundness. (F) Effect of K18 knockdown on the percentage of the luminal to the total (luminal+cells) area of the X-y cross-section of the tubule. In (C)-(F), data were analyzed as in Fig. 1. Data represent mean $\pm \mathrm{SD}$ from three independent experiments (at least 50 tubules per experiment). $* * * P<0.001$; n.s., not significant (one-way ANOVA followed by Dunnett's test).

was weaker than that in the latter. In contrast, in Soloknockdown cells, the ppMLC signal was markedly decreased throughout the tubule and its accumulation was slightly detected in the outer region, but not in the luminal region (Fig. 3A). As shown in Fig. 1B, F-actin predominantly accumulated in the luminal region in both control and Solo-knockdown tubules (Fig. 3A). To quantify these data, the fluorescence intensities of ppMLC and F-actin were analyzed by line profile analysis (Fig. 3B). Measurements of the relative fluorescence intensity of ppMLC and F-actin revealed that Solo knockdown significantly decreased the level of ppMLC in both the luminal and the basal regions of tubules (Fig. 3C), but had no apparent effect on the level of F-actin in both regions (Fig. 3D).

We next examined the effect of K18 knockdown on the levels of ppMLC and F-actin at the luminal and the basal region of MDCK tubules. Similar to the effects of Solo knockdown, K18 knockdown significantly decreased the level of ppMLC at both the luminal and basal tubule regions, but had no significant effect on the level of F-actin at either region (Fig. 4).

We also examined the effects of Solo or K18 knockdown on the levels of ppMLC and MLC in MDCK cells by immunoblot analyses. Knockdown of either Solo or K18 significantly decreased the level of ppMLC, whereas these treatments had no apparent effect on the level of total MLC (Supplementary Fig. S1).

Collectively, these results indicate that both Solo and $\mathrm{K} 18$ filaments are required for increasing or maintaining the ppMLC level in both the luminal and basal regions of MDCK tubules.

\section{Knockdown of Solo disorganizes K18 filaments}

We previously showed that Solo is required for proper organization of K8/K18 filaments in 2D-cultured MDCK cells (Fujiwara et al., 2016). To examine whether Solo is involved in the organization of $\mathrm{K} 8 / \mathrm{K} 18$ filaments in $3 \mathrm{D}-$ cultured epithelial tubules, we analyzed the effect of Solo knockdown on $\mathrm{K} 8 / \mathrm{K} 18$ filament organization in $3 \mathrm{D}$ - 
A

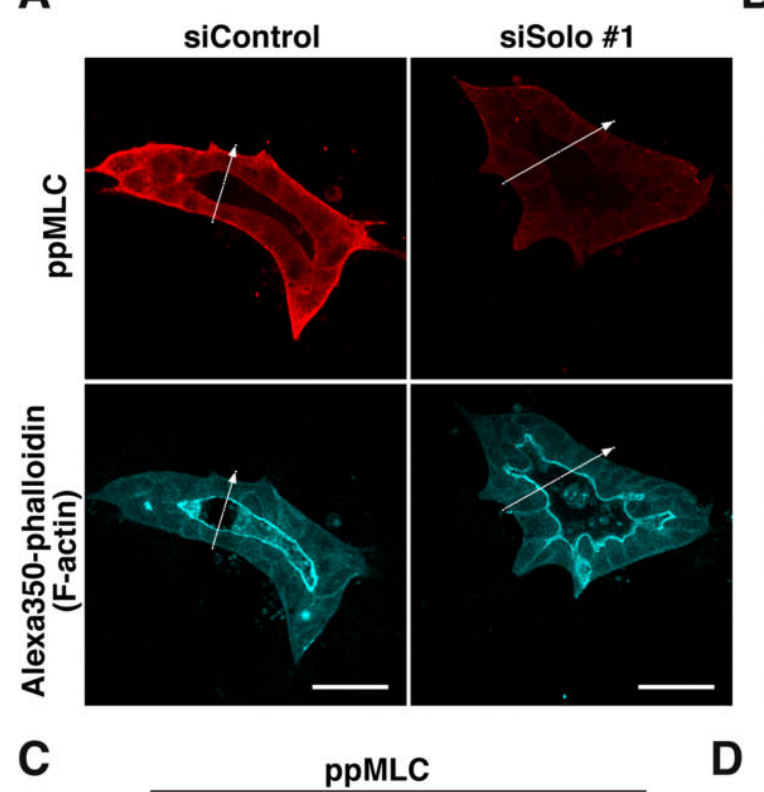

D
B sicontrol
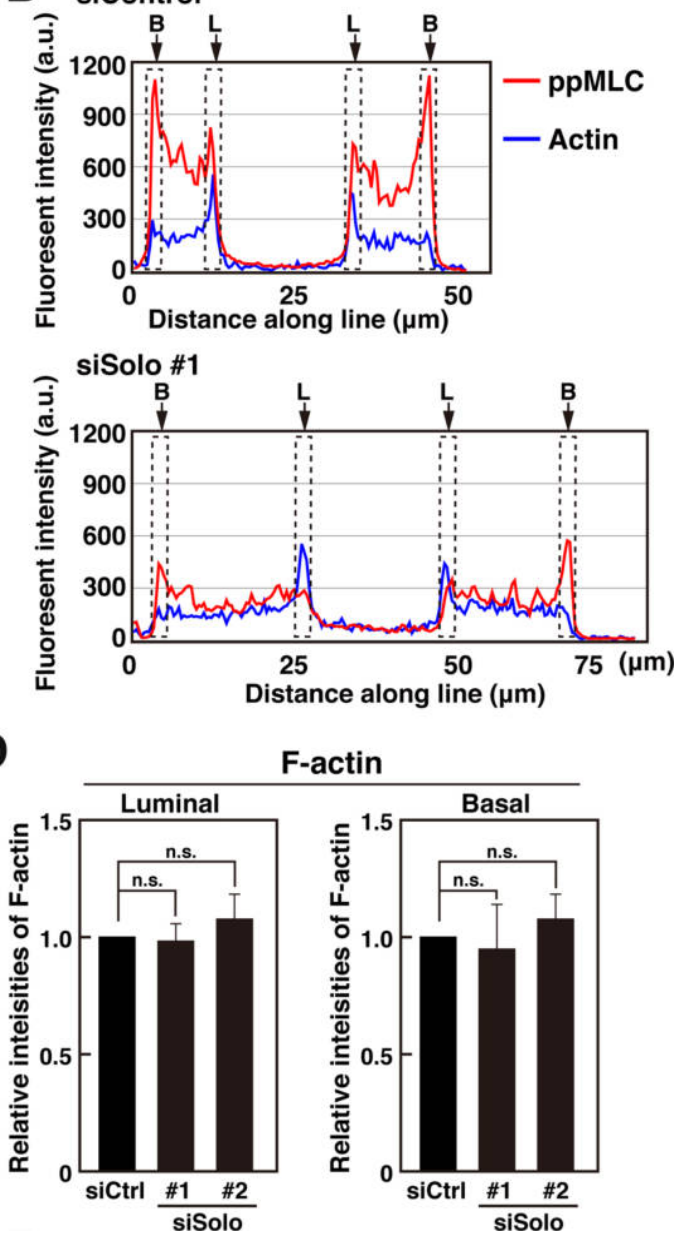

F-actin

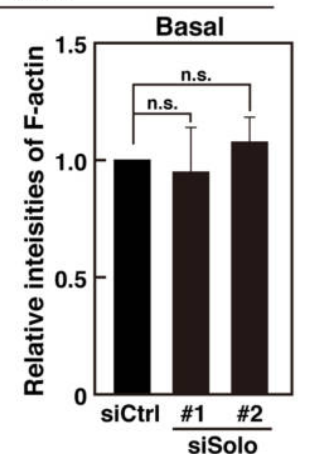

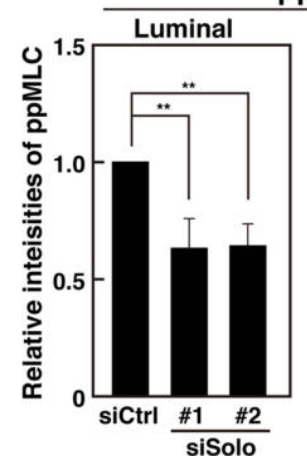

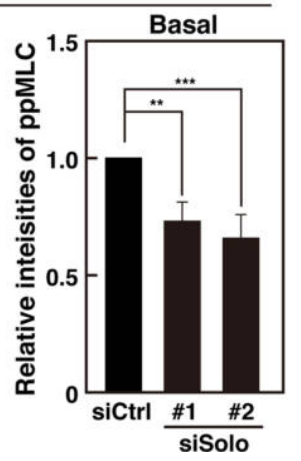

Fig. 3. Knockdown of Solo decreases the level of ppMLC at the luminal and the basal regions of tubules. (A) Representative images of F-actin (cyan) and ppMLC (red) in MDCK tubules. MDCK cells were treated with control or Solo-targeting siRNAs and cultured in 3D collagen gels for 4 days. The tubules were fixed and stained with Alexa 350-phalloidin and an anti-ppMLC antibody. Scale bar, $50 \mu \mathrm{m}$. (B) Line profiles of the fluorescence intensity of F-actin (blue) and ppMLC (red) along the white arrowed lines in (A). Arrows indicate the positions of the luminal (L) and the basal (B) region of tubules. All line profile analyses were performed using ImageJ software. (C, D) Quantification of the relative fluorescence intensity of ppMLC (C) and F-actin (D) at the luminal and the basal region of control and Solo-knockdown tubules. Relative intensities of ppMLC and F-actin are indicated with the values in control cells set to 1.0. Data represent mean $\pm \mathrm{SD}$ of three independent experiments (at least 10 tubules per experiment). ${ }^{* *} P<0.01 ; * * * P<0.001$; n.s., not significant (one-way ANOVA followed by Dunnett's test).

cultured MDCK tubules. To visualize the organization of K8/K18 filaments, we used MDCK cells stably expressing YFP-K18, and the tubules were analyzed by anti-GFP immunostaining of YFP-K18 and rhodamine-phalloidin staining for F-actin. In control cells, K18 filaments were well-organized and mostly oriented along the long axis of the tubules (Fig. 5A). In contrast, knockdown of Solo noticeably disrupted the orientation of K18 filaments (Fig. $5 \mathrm{~A}$ ). To quantitatively measure the orientation of K18 filaments, each tubule was fitted to an ellipsoid and the angle of orientation $(\theta)$ of K18 filaments against the long axis (x) of the ellipsoid was measured. Histogram analysis showed that K18 filaments aligned almost parallel to the long axis of tubules in control cells, but they were randomized in Solo-knockdown cells (Fig. 5B). Solo knockdown significantly reduced the percentage of K18 filaments oriented along the long axis of tubules $\left(-30^{\circ}<\theta<30^{\circ}\right)$ (Fig. 5C). We also examined the effect of Solo knockdown on the level of YFP-K18 protein by anti-GFP immunoblot analysis. Solo knockdown had no significant effect on the level of YFPK18 protein (Fig. 5D and 5E), suggesting that Solo has no effect on the stability of K18 protein. These results suggest that Solo is involved in the organization and proper orientation of K8/K18 filaments in epithelial tubules. 
A

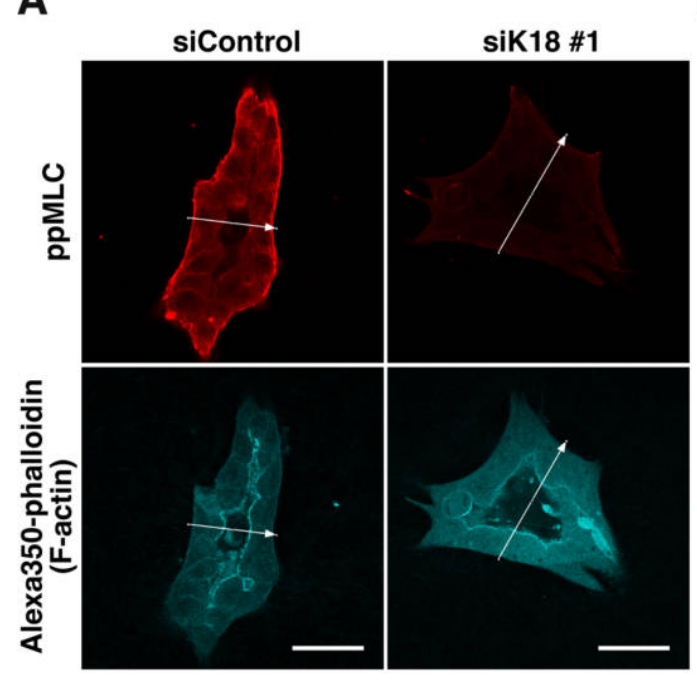

C

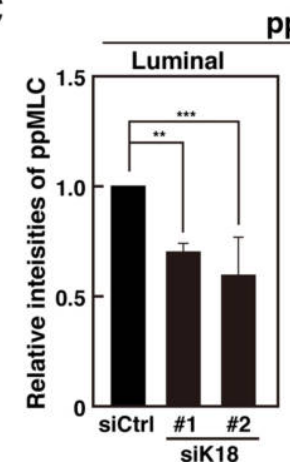

B

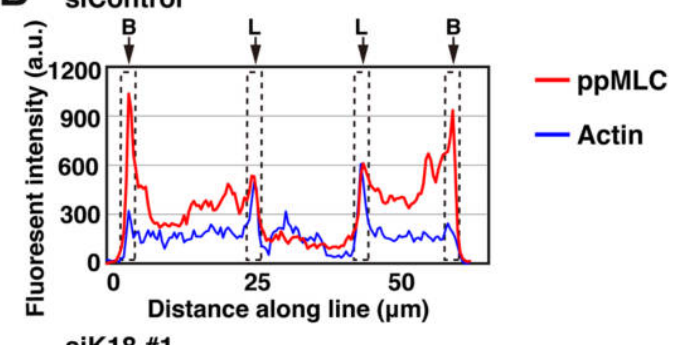

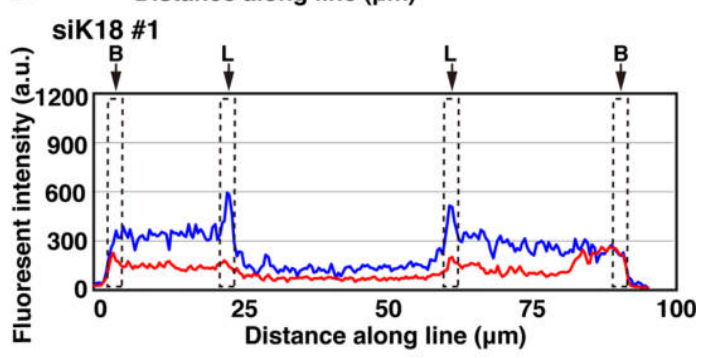

D

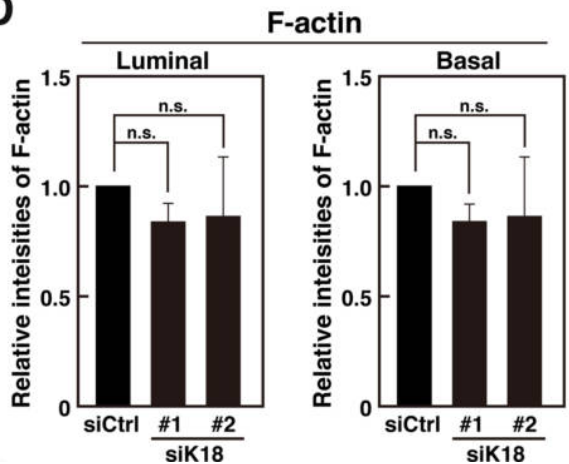

Fig. 4. Knockdown of K18 decreases the level of ppMLC at the luminal and the basal region of tubules. (A) Representative images of F-actin (cyan) and ppMLC (red) in control and K18-knockdown MDCK tubules. F-actin and ppMLC in tubules were visualized as in Fig. 3. Scale bar, $50 \mu \mathrm{m}$. (B) Line profiles of the fluorescence intensity of F-actin (blue) and ppMLC (red) along the white arrowed lines in (A). Arrows indicate the positions of the luminal (L) and the basal (B) region of tubules. (C, D) Quantification of the relative fluorescence intensity of ppMLC (C) and F-actin (D) at the luminal and the basal region of control and K18-knockdown tubules. Relative intensities of ppMLC and F-actin are indicated with the values in control cells set to 1.0. Data represent mean $\pm \mathrm{SD}$ of four independent experiments (at least 10 tubules per experiment). ${ }^{* *} P<0.01 ; * * * P<0.001$; n.s., not significant (one-way ANOVA followed by Dunnett's test).

\section{Discussion}

Solo is a RhoA-targeting GEF that was recently identified to be involved in tensional force-induced RhoA activation and stress fiber reinforcement with the aid of K8/K18 filaments (Abiko et al., 2015; Fujiwara et al., 2016); however, its physiological role has remained unknown. In this study, we showed that knockdown of either Solo or K18 resulted in rounder morphology and increased lumen size of 3Dcultured MDCK cells, indicating that Solo and K18 filaments play crucial roles in forming or maintaining the elongated morphology of epithelial tubules and limiting their lumen size. Knockdown of Solo or K18 also decreased the levels of the contractile force marker ppMLC in the luminal and outer walls of tubules, which likely suggests that Solo and K18 filaments are involved in elongated tubule morphogenesis by promoting or maintaining myosin II-mediated contractile force in the luminal and outer surfaces of tubules.

We previously demonstrated that Solo associates with K8/K18 filaments and that knocking down either Solo or K18 suppresses force-induced RhoA activation and stress fiber formation in 2D-cultured MDCK cells (Fujiwara et al., 2016). The suppressive effect of Solo knockdown on force-induced stress fiber formation was recovered by expressing wild-type Solo but not Solo mutants that do not localize to K8/K18 filaments (Fujiwara et al., 2016), suggesting that the association with $\mathrm{K} 8 / \mathrm{K} 18$ filaments is critical for the function of Solo in force-induced RhoA activation and stress fiber formation. In this study, we showed that similar to Solo knockdown, K18 knockdown in 3D-cultured MDCK tubules decreased the level of 
A
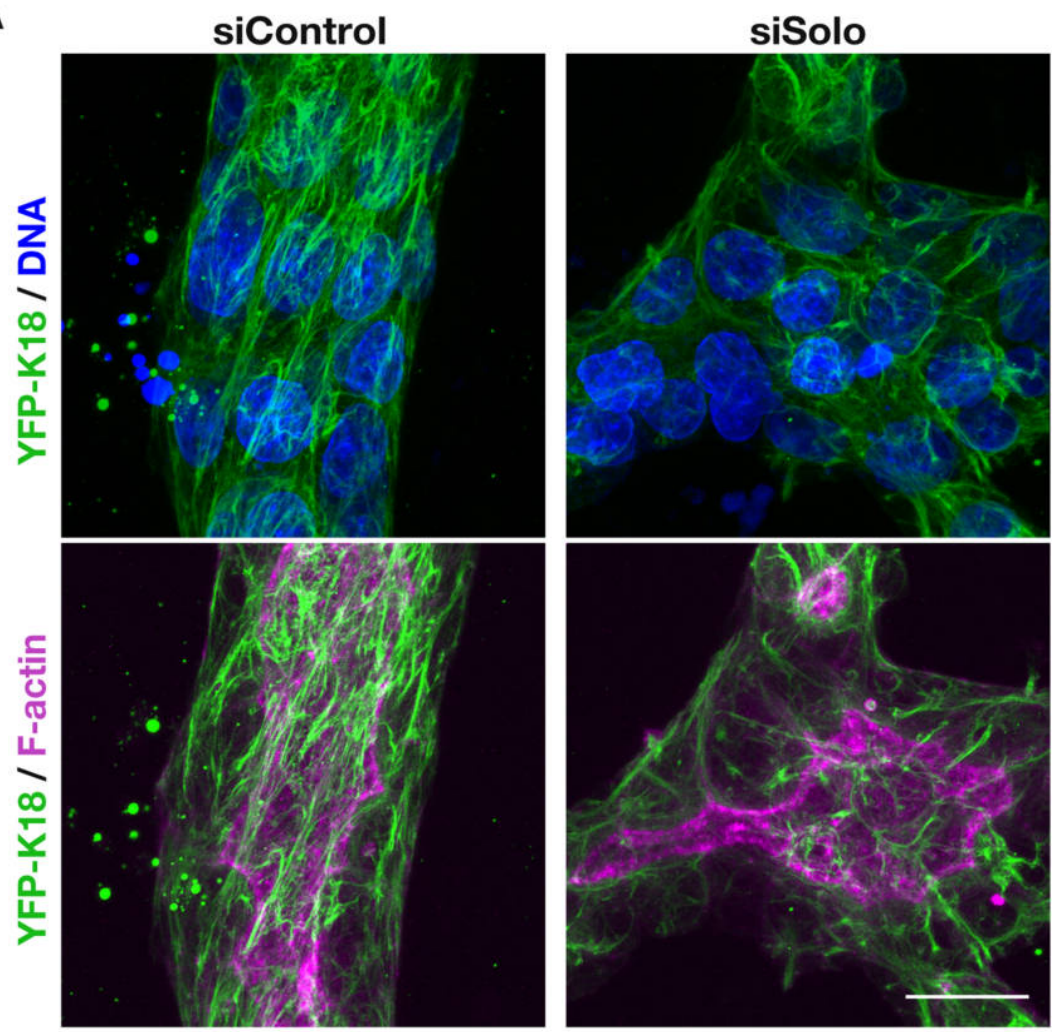

B

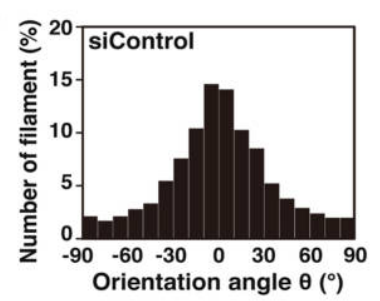

C

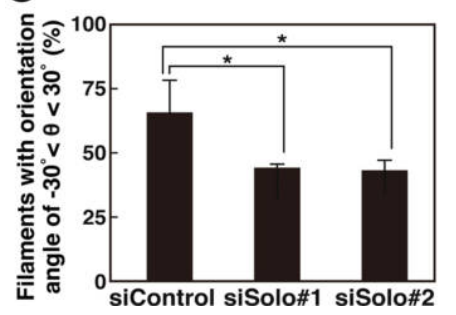

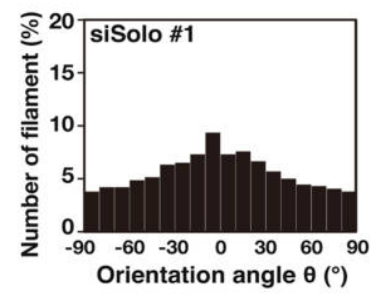

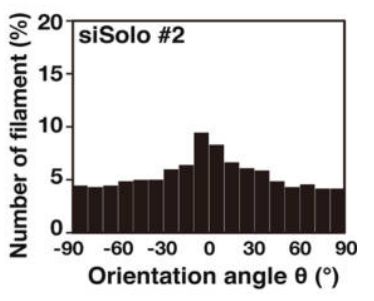

D

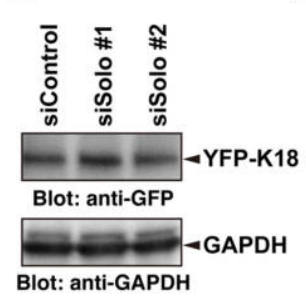

$\mathbf{E}$

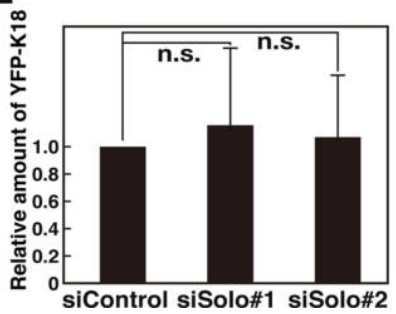

siSolo

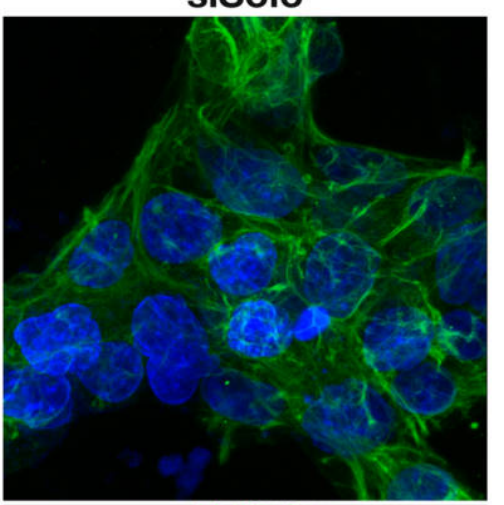

Fig. 5. Knockdown of Solo disorganizes K18 filaments. (A) Effect of Solo knockdown on the organization of K18 filaments. Representative z-stacked images of MDCK tubules. MDCK cells stably expressing YFP-K18 were transfected with either control or Solo-targeting siRNAs, cultured in 3D collagen gels for 4 days, and then fixed and immunostained with anti-GFP polyclonal antibodies for YFP-K18 (green), and stained with DAPI for nuclei (blue) and rhodamine-phalloidin for F-actin (magenta). Scale bar, $50 \mu \mathrm{m}$. (B) Effect of Solo knockdown on the orientation of K18 filaments. The angle ( $\theta$ ) of orientation of K18 filaments against the long axis of tubules was measured and the percentages of filaments with the indicated orientation angle were plotted in a histogram. (C) Quantitative analysis of the percentages of filaments with the orientation angle of $-30^{\circ}<\theta<30^{\circ}$. The data represent mean $\pm \mathrm{SD}$ of three independent experiments (at least 10 tubules per experiment). ${ }^{*} P<0.05$ (one-way ANOVA followed by Dunnett's test). (D) Effect of Solo knockdown on the level of YFP-K18. MDCK cells stably expressing YFP-K18 were transfected with either control or Solo siRNAs and cultured on plates for 2 days, and the cell lysates were analyzed by immunoblotting with anti-GFP and anti-GAPDH antibodies. (E) Quantitative analysis of the levels of YFP-K18 in control and Solo-knockdown cells. Data represent mean \pm SD of four independent experiments. n.s., not significant (one-way ANOVA followed by Dunnett's test). 
ppMLC and resulted in a rounder morphology. Thus, K8/K18 filaments are likely involved in elongated tubule morphology by assisting in Solo activation. On the other hand, knockdown of Solo or treatment with Y-27632, a selective inhibitor of ROCK, causes disorganization of $\mathrm{K} 8 / \mathrm{K} 18$ filaments in 2D-cultured MDCK cells (Fujiwara et al., 2016), indicating that the Solo-RhoA-ROCK pathway is required for the proper organization of $\mathrm{K} 8 / \mathrm{K} 18$ filaments. In this study, we also showed that Solo knockdown perturbs the orientation of K18 filaments in 3D-cultured MDCK tubules. These results suggest that K8/K18 filaments act not only as a regulator of Solo, but also as the downstream effector of the Solo-RhoA-ROCK pathway.

$\mathrm{K} 8 / \mathrm{K} 18$ filaments are the major keratin filaments in simple epithelia. We showed that K18 filaments are normally oriented along the long axis of tubules but are disorganized in the rounder tubules of Solo-knockdown cells and that knockdown of K18 results in rounder tubules, indicating that the proper organization of K18 filaments is crucial for the formation or maintenance of elongated tubule morphology. Considering the role of keratin filaments in providing mechanical support to cells, it is likely that the proper orientation of K18 filaments parallel to the long axis of the tubule contributes to maintain and stabilize the elongated morphology of epithelial tubules. Thus, K8/K18 filaments likely regulate epithelial tubule morphogenesis by assisting in the promotion of the Solo-RhoA pathway and by mechanically stabilizing the tubule morphology.

We showed that knockdown of Solo or K18 decreased the levels of ppMLC at the luminal and outer walls of tubules. Since Solo and K8/K18 filaments are involved in RhoA activation (Tse et al., 2005; Bordeleau et al., 2012; Abiko et al., 2015; Fujiwara et al., 2016), these proteins probably induce MLC phosphorylation through RhoA activation. Myosin II-mediated contractile forces appear to play critical roles in maintaining the elongated morphology of the tubule and narrowing the luminal size (Ferrari et al., 2008). A recent study showed that knockdown of p114RhoGEF (a RhoA-targeting GEF, also known as ARHGEF18) causes the formation of aberrant tubules with discontinuous and multiple lumens (Kim et al., 2015), suggesting that p114RhoGEF is required for consolidating multiple small lumens into a single lumen. In contrast, knockdown of Solo had no apparent effect on single lumen formation in tubules, suggesting that p114RhoGEF and Solo play distinct roles in tubulogenesis and that Solo plays a specific role in the formation or maintenance of elongated tubule morphology probably by generating the myosin II-mediated contractile force at the luminal and basal surfaces of the tubule and the longitudinally arranged K8/K18 filaments.

In summary, we showed that Solo plays a crucial role in the elongated morphology of epithelial tubules. Solo appears to be involved in generating myosin II-mediated contractile force at the cell periphery and promoting the proper organization of K18 filaments during epithelial tubule formation. It was reported that the zebrafish ortholog of Solo, Quattro, is involved in convergent extension cell movements of mesoderm cells during zebrafish gastrulation (Daggett et al., 2004). These findings suggest that Solo plays an important role in morphogenesis and homeostasis of various tissues. Further studies on the activation mechanism and physiological functions of Solo will provide new insights into how mechanical forces are transduced and involved in tissue morphogenesis and homeostasis.

Acknowledgments. We thank Dr. Shigenobu Yonemura (Tokushima University) for helpful comments and encouragement, Dr. Akira Kikuchi (Osaka University) and Dr. Hiroshi Koyama (National Institutes for Basic Biology) for helpful comments, and Yusuke Isozaki for technical assistance. This work was supported by Grants-in-Aid for Scientific Research from MEXT (grant number 23112005 and 16K07335 to K.O., and 15K14469 and 16H00749 to K.M.), Research Program on Advanced Research and Development Programs for Medical Innovation from the Agency for Medical Research and Development (16gm5810015h0001 to K.O.), Research Grants from the Uehara Memorial Foundation and the Japan Foundation for Applied Enzymology to K.M., and NIBB Collaborative Research Program (17-515 to K.K. and K.O.).

\section{References}

Abiko, H., Fujiwara, S., Ohashi, K., Hiatari, R., Mashiko, T., Sakamoto, N., Sato, M., and Mizuno, K. 2015. Rho guanine nucleotide exchange factors involved in cyclic-stretch-induced reorientation of vascular endothelial cells. J. Cell Sci., 128: 1683-1695.

Azuma, Y. and Onami, S. 2013. Evaluation of the effectiveness of simple nuclei-segmentation methods on Caenorhabditis elegans embryogenesis images. BMC Bioinformatics, 14: 295.

Bhadriraju, K., Elliott, J.T., Nguyen, M., and Plant, A.L. 2007. Quantifying myosin light chain phosphorylation in single adherent cells with automated fluorescence microscopy. BMC Cell Biol., 8: 43.

Bordeleau, F., Myrand Lapierre, M.E., Sheng, Y., and Marceau, N. 2012. Keratin 8/18 regulation of cell stiffness-extracellular matrix interplay through modulation of Rho-mediated actin cytoskeleton dynamics. PLoS One, 7: e38780.

Carpenter, A.E., Jones, T.R., Lamprecht, M.R., Clarke, C., Kang, I.H., Friman, O., Guertin, D.A., Chang, J.H., Lindquist, R.A., Moffat, J., Golland, P., and Sabatini, D.M. 2006. CellProfiler: image analysis software for identifying and quantifying cell phenotypes. Genome Biol., 7: R100.

Cook, D.R., Rossman, K.L., and Der, C.J. 2014. Rho guanine nucleotide exchange factors: regulators of Rho GTPase activity in development and disease. Oncogene, 33: 4021-4035.

Coulombe, P.A. and Wong, P. 2004. Cytoplasmic intermediate filaments revealed as dynamic and multipurpose scaffolds. Nat. Cell Biol., 6: 699-706.

Curtis, C., Hemmeryckx, B., Haataja, L., Senadheera, D., Groffen, J., and Heisterkamp, N. 2004. Scambio, a novel guanine nucleotide exchange factor for Rho. Mol. Cancer, 3: 10 .

Daggett, D.F., Boyd, C.A., Gautier, P., Bryson-Richardson, R.J., Thisse, C., Thisse, B., Amacher, S.L., and Currie, P.D. 2004. Developmentally restricted actin-regulatory molecules control morphogenetic cell movements in the zebrafish gastrula. Curr. Biol., 14: 1632-1638.

Ferrari, A., Veligodskiy, A., Berge, U., Lucas, M.S., and Kroschewski, R. 2008. ROCK-mediated contractility, tight junctions and channels contribute to the conversion of a preapical patch into apical surface during isochoric lumen initiation. J. Cell Sci., 121: 3649-3663.

Fujiwara, S., Ohashi, K., Mashiko, T., Kondo, H., and Mizuno, K. 2016. 
Interplay between Solo and keratin filaments is crucial for mechanical force-induced stress fiber reinforcement. Mol. Biol. Cell, 27: 954-966.

Fujiwara, S. and Mizuno, K. 2017. Role of intermediate filaments in cell locomotion. In: eLS. John Wiley \& Sons, Ltd: Chichester, a0026365.

Fukata, Y., Amano, M., and Kaibuchi, K. 2001. Rho-Rho-kinase pathway in smooth muscle contraction and cytoskeletal reorganization of nonmuscle cells. Trends Pharmacol. Sci., 22: 32-39.

Geiger, B., Spatz, J.P., and Bershadsky, A.D. 2009. Environmental sensing through focal adhesions. Nat. Rev. Mol. Cell Biol., 10: 21-33.

Goeckeler, Z.M. and Wysolmerski, R.B. 1995. Myosin light chain kinaseregulated endothelial cell contraction: the relationship between isometric tension, actin polymerization, and myosin phosphorylation. J. Cell Biol., 130: 613-627.

Hall, A. 2012. Rho family GTPases. Biochem. Soc. Trans., 40: 13781382.

Harada, K., Negishi, M., and Katoh, H. 2015. HGF-induced serine 897 phosphorylation of EphA2 regulates epithelial morphogenesis of MDCK cells in 3D culture. J. Cell Sci., 128: 1912-1921.

Hodge, R.G. and Ridley, A.J. 2016. Regulating Rho GTPases and their regulators. Nat. Rev. Mol. Cell Biol., 17: 496-510.

Hunter, M.P. and Zegers, M.M. 2010. Pak1 regulates branching morphogenesis in 3D MDCK cell culture by a PIX and beta1-integrindependent mechanism. Am. J. Physiol. Cell Physiol., 299: C21-C32.

Huveneers, S. and de Rooij, J. 2013. Mechanosensitive systems at the cadherin-F-actin interface. J. Cell Sci., 126: 403-413.

Iruela-Arispe, M.L. and Beitel, G.J. 2013. Tubulogenesis. Development, 140: $2851-2855$.

Kim, M., O’Brien, L.E., Kwon, S.H., and Mostov, K.E. 2010. STAT1 is required for redifferentiation during Madin-Darby canine kidney tubulogenesis. Mol. Biol. Cell, 21: 3926-3933.

Kim, M., Shewan, A.M., Ewald, A.J., Werb, Z., and Mostov, K.E. 2015. p114RhoGEF governs cell motility and lumen formation during tubulogenesis through a ROCK-myosin-II pathway. J. Cell Sci., 128: 43174327.

Lubarsky, B. and Krasnow, M.A. 2003. Tube morphogenesis: making and shaping biological tubes. Cell, 112: 19-28.

Marty-Santos, L. and Cleaver, O. 2016. Pdx1 regulates pancreas tubulogenesis and E-cadherin expression. Development, 143: 101-112.

Miao, H., Nickel, C.H., Cantley, L.G., Bruggeman, L.A., Bennardo, L.N., and Wang, B. 2003. EphA kinase activation regulates HGF-induced epithelial branching morphogenesis. J. Cell Biol., 162: 1281-1292.
Mizutani, T., Haga, H., Koyama, Y., Takahashi, M., and Kawabata, K. 2006. Diphosphorylation of the myosin regulatory light chain enhances the tension acting on stress fibers in fibroblasts. J. Cell Physiol., 209: 726-731.

Montesano, R., Schaller, G., and Orci, L. 1991a. Induction of epithelial tubular morphogenesis in vitro by fibroblast-derived soluble factors. Cell, 66: 697-711.

Montesano, R., Matsumoto, K., Nakamura, T., and Orci, L. 1991b. Identification of a fibroblast-derived epithelial morphogen as hepatocyte growth factor. Cell, 67: 901-908.

O’Brien, L.E., Zegers, M.M., and Mostov, K.E. 2002. Building epithelial architecture: insights from three-dimensional culture models. Nat. Rev. Mol. Cell Biol., 3: 531-537.

Ohashi, K., Fujiwara, S., and Mizuno, K. 2017. Roles of the cytoskeleton, cell adhesion and rho signalling in mechanosensing and mechanotransduction. J. Biochem., 161: 245-254.

Pollack, A.L., Runyan, R.B., and Mostov, K.E. 1998. Morphogenetic mechanisms of epithelial tubulogenesis: MDCK cell polarity is transiently rearranged without loss of cell-cell contact during scatter factor/ hepatocyte growth factor-induced tubulogenesis. Dev. Biol., 204: 64-79.

Recuenco, M.C., Ohmori, T., Tanigawa, S., Taguchi, A., Fujimura, S., Conti, M.A., Wei, Q., Kiyonari, H., Abe, T., Adelstein, R.S., and Nishinakamura, R. 2015. Nonmuscle myosin II regulates the morphogenesis of metanephric mesenchyme-derived immature nephrons. $J$. Am. Soc. Nephrol., 26: 1081-1091.

Sigurbjornsdottir, S., Mathew, R., and Leptin, M. 2014. Molecular mechanisms of de novo lumen formation. Nat. Rev. Mol. Cell Biol., 15: 665676.

Tse, S.W., Broderick, J.A., Wei, M.L., Luo, M.H., Smith, D., McCaffery, P., Stamm, S., and Andreadis, A. 2005. Identification, expression analysis, genomic organization and cellular location of a novel protein with a RhoGEF domain. Gene, 359: 63-72.

Wozniak, M.A. and Chen, C.S. 2009. Mechanotransduction in development: a growing role for contractility. Nat. Rev. Mol. Cell Biol., 10: 34 43.

Zegers, M.M., O’Brien, L.E., Yu, W., Datta, A., and Mostov, K.E. 2003. Epithelial polarity and tubulogenesis in vitro. Trends Cell Biol., 13: $169-176$.

(Received for publication, April 18, 2018, accepted, April 20, 2018 and published online, April 28, 2018) 